\title{
Modificación de la personalidad mediante una terapia cognitivo-conductual de afrontamiento al estrés
}

\author{
Julia Linares-Ortiz, Humbelina Robles-Ortega* y Maria I. Peralta-Ramírez
}

Departamento de Personalidad, Evaluación y Tratamiento Psicológico. Facultad de Psicología. Universidad de Granada (España)

\begin{abstract}
Resumen: El objetivo de este estudio ha sido comprobar la posibilidad de modulación de variables de personalidad (tales como algunos de los Cinco Grandes Factores, o la personalidad resistente) a través de la aplicación de un programa de afrontamiento del estrés. Para ello, han participado 26 personas del ámbito universitario con alto estrés percibido, distribuidas en dos grupos (grupo de tratamiento y grupo de control). Los instrumentos de evaluación seleccionados se clasificaron en dos grupos: variables psicológicas y emocionales y variables de personalidad. Los resultados encontrados mediantes un ANOVA de medidas repetidas mostraron que existía interacción momento $\mathrm{x}$ grupo en las variables optimismo, extraversión y responsabilidad, correspondientes al Modelo de los Cinco Grandes Factores, mostrando un incremento de las puntuaciones en estas variables en el grupo terapia y no encontrándose dicha interacción en el grupo control. Las puntuaciones en los componentes de neuroticismo, amabilidad, apertura a la experiencia y personalidad resistente no se modificaron en ninguno de los dos grupos.

Este estudio apoya la idea de que modificando determinados parámetros emocionales relacionados con el estrés mediante terapia cognitivoconductual se pueden ver modulados algunos factores de personalidad.

Palabras clave: Estrés; terapia cognitivo-conductual; personalidad resistente; Cinco Grandes Factores; personalidad.
\end{abstract}

Title: Personality modification by a cognitive-behavioral therapy of stress coping.

Abstract: The objective of this study was to assess the possibility of varying personality variables (such as some of the Big Five factors, or hardiness) through the application of a stress doping therapy. Twenty-six people participated from universities with high perceived stress, divided into two groups (treatment group and control group). The assessment instruments selected were classified into two groups: psychological and emotional variables and personality variables. The results showed that after the program, the experimental group showed a decline in scores of stress, psychopathology and worries. On the other hand, personality variables, interaction was found in the variables moment $\mathrm{x}$ group, optimism, extraversion factor and accountability, corresponding to the model of the Big Five factors showed increased scores on these variables. However, these changes were not in the control group. The hardy personality scores did not change in either group. This study tests the modulation of certain factors of personality through cognitive-behavioral therapy for stress management, after which beneficial effects are obtained.

Key words: Stress; cognitive-behavioral therapy; hardiness personality; Big Five Factors; personality.

\section{Introducción}

En la actualidad, el estrés psicológico es una de las variables más estudiadas debido a las consecuencias que éste tiene tanto a nivel físico como psicológico (Ho, Neo, Chua, Check y Mak, 2010; Sapolsky, 2008). La investigación actual señala que, en nuestra sociedad, el estrés tiene graves repercusiones tanto en el nivel socioeconómico (en términos de absentismo y pérdida de productividad) como de sufrimiento humano. La cada vez mayor frecuencia, intensidad y variedad del tipo de demandas que genera el estilo de vida occidental, activan respuestas de estrés que pueden resultar dañinas para nuestra salud. Éste es uno de los problemas con el que nos enfrentamos en el siglo XXI. De ahí la relevancia de los esfuerzos dirigidos a profundizar en cómo controlar sus efectos negativos (Robles Ortega y Peralta Ramírez, 2006).

Numerosos estudios han mostrado que mediante terapias cognitivo-conductuales se consigue controlar el estrés, así como variables asociadas a éste: ansiedad (Hofmann y Smits, 2008; Navarrete-Navarrete et al., 2010; Öst, 2008; Peralta-Ramírez, Robles-Ortega, Navarrete-Navarrete y Jiménez-Alonso, 2009), depresión (Navarrete-Navarrete et al., 2010; Peralta-Ramírez et al., 2009; Spangler, Simons, Monroe y Thase, 1997), felicidad (Lyubomirsky, King y Diener, 2005; Schiffrin y Nelson, 2010), sentido del humor (Grases,

\footnotetext{
* Dirección para correspondencia [Correspondence address]: Humbelina Robles Ortega. Departamento de Personalidad, Evaluación y Tratamiento Psicológico. Facultad de Psicología. Campus de Cartuja s/n. Universidad de Granada. 18071 Granada (España).

E-mail: hrobles@ugr.es
}

Trías, Sánchez y Zárate, 2010), optimismo (Carver y Scheier, 2002; Shifren y Hooker, 1995), somatizaciones, hostilidad (López, Kasanzew y López, 2007), entre otras.

Debido al fuerte impacto que este trastorno puede llegar a suponer en las personas, se han desarrollado programas en diferentes muestras de población tratando de paliar dichas consecuencias (Navarrete-Navarrete et al., 2010; PeraltaRamírez, et al., 2009; Robles Ortega y Peralta Ramírez, 2006). En concreto, Butler, Chapman, Forman y Beck (2006) examinaron 16 meta-análisis de los efectos de la terapia cognitivo-conductual y encontraron mejoras en una gran variedad de problemas entre los que destacaban depresión mayor, trastorno de ansiedad generalizada y trastorno de estrés post-traumático.

Existen diferentes planteamientos teóricos que intentan explicar por qué se estresan los seres humanos y por qué unas personas se estresan más que otras. Uno de estos planteamientos define el estrés como un proceso, proceso en el que existe una relación particular entre el individuo y su entorno (Lazarus y Folkman, 1986). Y en esta relación se ha observado la incidencia de una serie de variables que pueden funcionar como protectoras, o como elicitadoras del estrés, variables tales como el apoyo social (Sandín, 2003), el estilo de afrontamiento (Hombrados, 1997), la predisposición biológica (Sapolsky, 2008), variables relacionadas con la toma de decisiones (Santos-Ruiz et al., 2012) y las variables personales o disposicionales (Denollet, et al., 2009; Padilla Montemayor, Peña Moreno y Arriaga González, 2006; Riolli y Savicki, 2003). Entre las variables predisposicionales relacionadas con el estrés, se ha estudiado ampliamente el optimismo, la personalidad tipo A y la personalidad resistente. 
Probablemente este último ha sido uno de los más estudiados en relación con el estrés. El constructo de resistencia fue desarrollado por Kobasa (1979), dentro de la teoría existencial, definiéndolo de la siguiente manera: "la actitud de una persona ante su lugar en el mundo que expresa simultáneamente su compromiso, control y disposición a responder ante los retos" (Kobasa, 1979 , p. 2). Los estudios realizados con el constructo de personalidad resistente muestran su relevancia como variable mediadora en los procesos de estrés y salud (Funk, 1992; Kobasa, 1982; Kobasa, Maddi y Kahn, 1982; Moreno Jiménez, Morett Natera, Rodríguez Muñoz y Morante Benadero, 2006; Ríos Rísquez, Godoy Fernández y Sánchez-Meca, 2011). En esta línea los resultados encontrados indican que las puntuaciones altas en personalidad resistente se asocian a menores síntomas asociados. Según Kobasa (1982), los individuos con personalidad resistente se enfrentan de forma activa y comprometida a los estímulos estresantes, percibiéndolos como menos amenazantes. Por otro lado, subrayar que el optimismo disposicional está ampliamente relacionado con la percepción de estrés (Nes y Segerstrom, 2006). Por último, recientes estudios han encontrado que el modelo de los Cinco Grandes Factores de Personalidad propuesto por Costa y McCrae (1992) también se relaciona ampliamente con el estrés y más en concreto con la supresión inmunológica que éste produce modulando la producción de células natural killer y células B (Ožura, Ihan y Musek, 2012).

De este modo personalidad y estrés están ampliamente relacionados, fundamentalmente por: (1) la elección o la evitación de los ambientes que están asociados con factores estresantes específicos, retos o beneficios, (2) la forma de interpretar una situación de estrés y la evaluación de las propias habilidades y capacidades para la conducta proactiva de uno con el fin de enfrentar o evitar, (3) la intensidad de la respuesta a una situación estresante, y (4) las estrategias de afrontamiento utilizadas por el individuo frente a una situación estresante (Lecic-Tosevski, Vukovic y Stepanovic, 2011). En concreto, algunos estudios han registrado una considerable consistencia en las estrategias empleadas para hacer frente a situaciones de estrés, independientemente de los factores situacionales y en relación con la personalidad permanente y rasgos temperamentales, como neuroticismo, extraversión, el sentido del humor, la persistencia, el fatalismo, la conciencia, y apertura a nuevas experiencias. Sin embargo, incluso las personas con personalidades maduras e integradas expuestas a estrés prolongado pueden experimentar el fracaso de sus capacidades de adaptación y descompensación psíquica o somática.

A pesar de esta clara relación no se han realizado estudios destinados a comprobar si se pueden modificar factores de personalidad muy relacionados con el estrés y su respuesta mediante una terapia de corte cognitivo conductual. Por ello, el objetivo de nuestro estudio ha sido comprobar si mediante una terapia de afrontamiento al estrés se pueden modificar diferentes factores de personalidad muy relacionados con éste como es la personalidad resistente, el optimismo y los 5 grandes factores de personalidad.

\section{Método}

\section{Muestra}

En este estudio participaron 30 personas, sin embargo completaron el estudio hasta el final 26 personas que fueron distribuidas en dos grupos, 13 en el grupo de tratamiento y 13 en el grupo control. Todos ellos respondían a un perfil similar: profesor/a, estudiante de postgrado (Máster o doctorado), o Personal de Administración y Servicios (PAS) de la Universidad de Granada y Córdoba. Los criterios de inclusión fueron: saber leer y escribir, presentar puntuaciones superiores a la media en estrés percibido y no presentar ningún tipo de psicopatología que pudiera interferir en los resultados del estudio. Las 4 personas que no completaron el estudio fueron 1 del grupo experimental por no completar el $80 \%$ de las sesiones (para la consideración de los datos de postratamiento del grupo experimental, se exigió que asistieran al menos al 80\% de las sesiones), y 3 del grupo control que no participaron en la evaluación "pos-tratamiento".

El grupo de tratamiento estuvo formado por ocho mujeres y cinco hombres. El rango de edad se encontraba entre 26 y 65 años, siendo la media de 42.92 años $(D T=10.07)$. Seis miembros del grupo estaban solteros y siete casados. Dos miembros del grupo eran de origen sudamericano y once de nacionalidad española. Por otro lado, el grupo control fue igualado al grupo terapia en las principales variables sociodemográficas: este grupo estaba también constituido por 5 hombres y 8 mujeres. El rango de edad oscilaba entre 27 y 57 , con una media de 46.17 años $(D T=8.91)$. Siete miembros del grupo control estaban solteros y seis casados. Igualmente, dos miembros del grupo eran de origen sudamericano y once de nacionalidad española. Tanto el grupo experimental como en el grupo control estaba compuesto por 6 profesores, 4 PAS y 3 estudiantes de posgrado.

\section{Diseño}

Se utilizó un diseño mixto de grupos independientes con medidas repetidas. Los grupos independientes fueron el grupo experimental y el grupo control. Las medidas repetidas fueron las variables psicológicas y emocionales y variables de personalidad obtenidas antes de la aplicación de la terapia de afrontamiento al estrés (pre-tratamiento) y una vez finalizada la terapia (pos-tratamiento).

\section{Instrumentos}

Los instrumentos utilizados para la evaluación pretratamiento y pos-tratamiento fueron los siguientes:

Variables psicológicas y emocionales

- Escala de Estrés Percibido. (Cohen, Kamarak y Mermeistein, 1983). Se administró la versión española de Remor y Carrobles, 2001. Se trata de un autoinforme que evalúa el 
grado en que las situaciones de la vida se perciben como estresantes. Consta de 14 ítems con cuatro alternativas de respuesta (0: nunca a 4: muy a menudo), siendo 56 la puntuación máxima En la adaptación española se obtuvieron valores óptimos de fiabilidad (consistencia interna, $a=.81$, y test-retest, $r=.73$ ), validez concurrente, y sensibilidad (Remor, 2006).

- Inventario de Sintomas, SCL-90-R (Derogatis, 1994). Se administró la versión española de De las Cuevas, González de Rivera, Henry y Gracia (1991). Este autoinforme está compuesto por 90 ítems, que se puntúan de 0 (nada en absoluto) a 4 (mucho o extremadamente). Evalúa nueve dimensiones primarias de malestar psicológico (somatizaciones, obsesiones y compulsiones, sensibilidad interpersonal, depresión, ansiedad, hostilidad, ansiedad fóbica, ideación paranoide y psicoticismo), así como tres índices globales: índice de severidad global (IGS), total de síntomas positivos (SP) y malestar sintomático positivo (PSDI). Este instrumento dispone de una fiabilidad y validez satisfactorias cuyo alfa de Cronbach para cada una de las subescalas es la siguiente: somatización, .90; obsesivo-compulsivo, .85; sensibilidad interpersonal, .85; depresión, .91; ansiedad, .86; hostilidad, .83; ansiedad fóbica, .90; síntomas paranoides, .64; síntomas psicóticos, 86 .

- Inventario de Vulnerabilidad al Estrés (Beech, Burns y Scheffield, 1986), en versión española de Robles-Ortega, Peralta-Ramírez y Navarrete-Navarrete (2006). Mide la predisposición del individuo a verse influido por el estrés percibido. Este instrumento consta de 22 ítems de respuesta dicotómica (si o no) donde la máxima puntuación que se puede obtener es 22. A mayor puntuación mayor vulnerabilidad al estrés. En relación a la consistencia interna, presenta un alfa de Cronbach $a=.87$ y una alta validez convergente con el Inventario de Ansiedad-Rasgo (STAI-R) $(r$ $=.595)$, el Inventario de Depresión de Beck (BDI) $(r=$ .647), la Escala de Síntomas Somáticos-Revisada (ESS-R) $(r=.613)$ y la Escala de Experiencias Vitales Recientes (SRLE) $(r=.496)$.

- Inventario de Preocupación, PSQW (Meyer, Miller, Metzger y Borkover, 1990) en versión española de Sandín-Ferrero, Chorot-Rasgo, Valiente-García y Lostao-Unzu (2009). Este instrumento evalúa el grado de preocupación permanente así como el componente cognitivo de las respuestas de ansiedad. Está constituido por 16 ítems, cuya puntuación va desde 1 (nada característico de mî) a 5 (muy característico de mî); a mayor puntuación corresponde mayor nivel de preocupaciones. La validación inicial se realizó en una muestra de estudiantes, y presentó una consistencia interna entre .93 y .94. En el trabajo posterior llevado a cabo por Brown, Antony y Barlow (1992) con pacientes con trastornos de ansiedad, obtuvo una consistencia interna de .95. En lo referente a la estabilidad temporal obtenida con el test-retest oscila, en varios estudios, entre .74 y .92 (Meyer et al., 1990).

\section{Variables de personalidad}

- NEO Five Factor Inventory, NEO-FFI (Costa y McCrae, 1992) validado en versión española por Manga, Ramos y Morán (2004). Este cuestionario consta de 60 ítems, los cuales han de responderse sobre una escala tipo Likert pudiendo puntuar cada ítem en una escala de 1 a 5 que va de A ("En total desacuerdo") a E ("Totalmente de acuerdo"). Fue desarrollado con el objetivo de dar una medida más concisa de los cinco factores básicos de la personalidad: Neuroticismo (N), Extraversión (E), Apertura a la experiencia (O), Amabilidad (A) y Consciencia/Responsabilidad (C). Las consistencia interna (alfa de Cronbach) de sus escalas es la siguiente: Factor N, $a=.82$; factor E, $a$ $=.81$; factor $\mathrm{O}, a=.76$; factor $\mathrm{A}, a=.71$; factor $\mathrm{C}, a=$ .81 .

Life Orientation Test, LOT-R (Scheier, Carver y Bridges, 1994). Versión española de Ferrando, Chico y Tous (2002). Evalúa la dimensión de optimismo disposicional a través 10 ítems representados en una escala tipo Likert que oscila de 0 (estoy totalmente en desacuerdo) a 4 (estoy totalmente de acuerdo). Los diversos estudios sobre la fiabilidad de este cuestionario muestran índices que oscilan entre $a=.74$ (Schou, Ekeberg, Ruland, Sandwik, y Karensen, 2004) у $a=.75$ (Martínez-Correa, Reyes del Paso, GarcíaLeón y González-Jareño, 2006).

- Cuestionario de Personalidad Resistente, (CPR) (MorenoJiménez, Garrosa Hernández y González Gutiérrez, 2000). Este instrumento consta de 21 ítems, los cuales miden tres dimensiones: compromiso o implicación, reto y control. El rango de respuesta oscila entre 1 (totalmente en desacuerdo) y 4 (totalmente de acuerdo). Así pues, las puntuaciones son directamente proporcionales, es decir, puntuaciones más altas serían indicativas de una óptima personalidad resistente. Los datos de fiabilidad son aceptables (alfa de Cronbach de .74, .79 y .83 para Control, Compromiso y Reto respectivamente).

\section{Procedimiento}

El proceso de reclutamiento que se siguió con el grupo experimental fue el siguiente. En primer lugar, se mandó, desde la Clínica de Psicología de la Facultad de Psicología de la Universidad de Granada, un e-mail a todos los miembros de la Universidad (profesores, alumnos y PAS) ofertando la oportunidad de formar parte de un programa para el control del estrés. Pasadas dos semanas, se citaron a todas aquellas personas que habían solicitado participar en el programa realizándoles una entrevista inicial individual, en la cual también se administró el EEP con el fin de obtener la información necesaria para valorar si reunían los criterios de inclusión establecidos en la investigación. Además, tras informarles de este estudio y firmar el consentimiento informado se hizo entrega del cuadernillo con los cuestionarios pertenecientes a la pre-evaluación (línea base). Los cuestionarios que se administraron tanto en la fase pre-tratamiento como en la fase 
pos-tratamiento han sido todos los descritos en el apartado anterior de Instrumentos.

Finalmente, todos los solicitantes que reunían los criterios de inclusión pasaron a formar parte del programa distribuyéndose en dos grupos (cada uno de ellos con un horario diferente para facilitar la asistencia), en función de su disponibilidad horaria.

La selección de personas que componían el grupo control se realizó mediante el procedimiento de bola de nieve. Es decir, se pidió a las personas pertenecientes al grupo experimental que propusieran participar en el estudio a personas de su entorno con perfiles similares al suyo que manifestaran quejas de altos niveles de estrés. Se les instruyó para que dijeran a su posible control que se trataba de participar en un estudio sobre aspectos psicológicos de las personas que sufren estrés. Y se les ofrecía la posibilidad si estaban interesados de asistir al taller afrontamiento al estrés en el curso siguiente (podemos decir que el grupo control era un grupo en lista de espera).

Una vez seleccionados, se hizo entrega a cada uno de los participantes de un documento informativo y tras firmar el consentimiento informado pasaron a ser evaluados psicológicamente. El diseño y aplicación de esta investigación ha seguido fielmente todas las leyes elaboradas en la Declaración de Helsinky.

La terapia, de tipo cognitivo-conductual (Robles Ortega y Peralta Ramírez, 2006), fue impartida por dos psicólogas con amplia experiencia en este campo. Así pues, a lo largo de 14 sesiones, se trabajaron los siguientes componentes: 1) Conceptualización del estrés; 2) Técnicas de desactivación (I): Respiración diafragmática y entrenamiento autógeno; 3) Técnicas de desactivación (II): Relajación muscular profunda y técnicas de imaginación temática; 4) Técnicas cognitivas: Reestructuración cognitiva (I): Distorsiones o errores de pensamiento; 5) Técnicas cognitivas: Reestructuración cognitiva (II): Ideas irracionales; 6) Técnicas cognitivas: Reestructuración cognitiva (III): Debatiendo los pensamientos; 7) Otras técnicas cognitivas: a) Autoinstrucciones, y b) Parada del Pensamiento; 8) Técnicas asertivas (I): Qué es el comportamiento asertivo. Derechos asertivos básicos; 9) Técnicas asertivas (II): a) Cómo decir no, y B) Cómo pedir un cambio de conducta; 10) Técnicas de administración y control del tiempo; 11) Características de personalidad y su relación con el estrés y la salud (I): Personalidad tipo A, personalidad tipo $\mathrm{C}$ y personalidad tipo $\mathrm{R}$; 12) Características de personalidad y su relación con el estrés y la salud (II): control de la ira/hostilidad; 13) Humor y optimismo, y 14) Integración de los contenidos desarrollados a lo largo del programa.

El proceso que se siguió en todas las sesiones fue el mismo: comentar y recoger las tareas mandadas para casa la semana anterior, exposición de los contenidos correspondientes a la sesión semanal, práctica mediante rol-play de los contenidos expuestos y entrega de nuevas tareas. Las tareas consistieron en la mayoría de las ocasiones en el autorregistro y entrenamiento de los componentes explicados. Este tratamiento se lleva a cabo en grupo, cada grupo están compuestos por unas 6 o 7 personas. Cada sesión es semanal y su duración aproximada es de entre 1.30 y 2 horas. Son 14 sesiones por lo que el tratamiento completo dura tres meses y medio. Este tratamiento se llevó a cabo en los Servicios de Atención Psicológica de la Facultad de Psicología de la Universidad de Granada (actualmente, Clínica de Psicología de la Universidad de Granada).

Una vez finalizadas las 14 sesiones, se volvió a hacer entrega de los mismos cuestionarios de la pre-evaluación, con el fin de obtener las puntuaciones correspondientes a la post-evaluación. En lo referente al grupo control, el proceso de reclutamiento fue a través de los propios participantes en el programa y del propio entorno de los investigadores. Así, al principio del programa, se les pidió que entregaran el cuadernillo con los cuestionarios a aquellas personas de su entorno que presentaran un perfil similar al suyo, en lo que a variables sociodemográficas se refería (edad, estado civil, profesión, etc.). Una vez finalizado el programa, se volvió a hacer entrega del cuadernillo para que lo hicieran llegar de nuevo a los participantes del grupo control.

\section{Análisis estadístico}

Dado que en las variables estudiadas se cumplían los criterios de normalidad y homocedasticidad (homogeneidad de las varianzas) todos los análisis estadísticos realizados han sido con análisis paramétricos.

En primer lugar para comprobar si estaban igualadas las características basales de los participantes de ambos grupos, se realizaron diferentes $t$ de Student y $\chi^{2}$ siendo la variable independiente el grupo con dos niveles (grupo experimental y grupo control) y las variables dependientes las puntuaciones en las diferentes variables sociodemográficas, emocionales y de personalidad.

En segundo lugar para comprobar el efecto de la terapia sobre las variables descritas realizamos sucesivos análisis estadísticos. En primer lugar, realizamos un análisis de varianza univariado mixto $2 \mathrm{x}(2)$, para cada una de las variables analizadas, teniendo el primer factor entre grupos independientes dos niveles (grupo tratamiento y grupo control) y un segundo factor intrasujeto de medidas repetidas con dos niveles (momento basal y momento post-terapia). Las variables dependientes fueron las puntuaciones de percepción de estrés, síntomas psicopatológicos, personalidad (neuroticismo, extraversión, amabilidad, apertura a la experiencia y conciencia/responsabilidad) personalidad resistente, optimismo, vulnerabilidad al estrés y preocupaciones. En el análisis de medidas repetidas se aplicó la corrección de Greenhouse-Geisser. Por último, en el caso de las variables en las que existía interacción momento $\mathrm{x}$ grupo, con el objetivo de comprobar si existían diferencias significativas entre el momento inicial del estudio y el momento posterior, en los participantes de cada uno de los dos grupos (intrasujeto) se realizaron diferentes $t$ para muestras relacionadas en las distintas variables registradas. 


\section{Resultados}

\section{Análisis descriptivo de la muestra}

- Variables Sociodemográficas. En primer lugar, se procedió a comprobar si los grupos estaban igualados en las principales variables sociodemográficas. Para ello se realizaron diferentes $t$ de Student, siendo la variable independiente (VI), el grupo (tratamiento vs. control) y las variables dependientes (VD), la edad, nivel de estudios y número de hijos. Los resultados mostraron que no existían diferencias estadísticamente significativas para las variables edad $(t=$ $.85 ; p=.404)$, nivel de estudios $(t=.687 ; p=.50) \mathrm{y} \mathrm{n}^{\circ} \mathrm{de}$ hijos $(t=-1.141 ; p=.265)$. También se realizaron diferentes $\chi^{2}$ para las variables cualitativas, con respecto a la profesión y el estado civil, no observándose diferencias significativas entre ambos grupos, $\left(\chi^{2}=2.33 ; p=.506\right.$ y $\chi^{2}=$ 2.667; $p=.264$, respectivamente).

- Variables psicológicas y de personalidad. Para comprobar si existían diferencias entre los dos grupos en las principales variables psicológicas y de personalidad al comienzo del estudio se realizaron diferentes $t$ de Student. Los resultados encontrados mostraron que ambos grupos (grupo tratamiento y grupo control) estaban igualados en estrés percibido, vulnerabilidad al estrés, optimismo, los 5 factores de personalidad (neuroticismo, extraversión, apertura a la experiencia, amabilidad y consciencia/responsabilidad), las tres dimensiones de personalidad resistente (implicación, reto y control) y las subescalas del SCL-90 denominadas somatización, obsesión compulsión, depresión y ansiedad fóbica. Sin embargo los resultados mostraron que existían diferencias estadísticamente significativas entre los dos grupos en las subescalas del SCL-90 denominadas sensibilidad interpersonal $(t=2.53 ; p=.018)$, ansiedad $(t=2.49$; $p=.02)$, hostilidad $(t=2.23 ; p=.03)$, ideación paranoide $(t$ $=2.49 ; p=.02)$ y psicoticismo $(t=2.20 ; p=.03)$ siendo mayores las puntuaciones del grupo tratamiento que las del grupo control en estas variables. Así mismo, también encontramos diferencias entre el grupo control y el grupo tratamiento en la variable preocupaciones $(t=3.32 ; p=$ $.003)$ siendo mayores las puntuaciones de este último.

\section{Eficacia del la terapia cognitivo-conductual para el control del estrés}

- Modificación de variables psicológicas y emocionales. En primer lugar, realizamos un Análisis de Varianza univariado mixto de medidas repetidas $2 \mathrm{x}(2)$, para cada una de las variables analizadas, teniendo el primer factor entre grupos independientes dos niveles (grupo terapia y grupo control) y un segundo factor intrasujeto de medidas repetidas con dos niveles (momento basal y momento post-terapia). Las va- riables dependientes fueron las puntuaciones de percepción de estrés, síntomas psicopatológicos, vulnerabilidad al estrés y preocupaciones. En los factores de medidas repetidas se aplicó la corrección de Greenhouse-Geisser. Los resultados mostraron que existían interacción entre momento $\mathrm{x}$ grupo en las siguientes variables: estrés percibido $[F(1,25)=10.79 ; p=.003]$; vulnerabilidad al estrés $[F(1,25)$ $=5.83 ; p=.024]$; preocupaciones $[F(1,25)=12.85 ; p=$ $.001]$; somatizaciones $[F(1,25)=5.41 ; p=.029]$; sensibilidad interpersonal $[F(1,25)=6.65 ; p=.016]$; depresión $[F(1,25)=7.41 ; p=.012]$; ansiedad $[F(1,25)=14.15 ; p=$ $.001]$ y ansiedad fóbica $[F(1,25)=24.00 ; p=.000]$. No se encontró interacción momento x grupo en las demás variables.

En segundo lugar, en las variables en las que existía interacción se llevaron a cabo diferentes $t$ de Student para muestras relacionadas; con ello se pretendía comprobar si había diferencias entre el momento preterapia y posterapia en cada uno de los dos grupos. Los resultados encontrados se recogen en la Tabla 1.

- Modificación de variables de personalidad. Por otro lado, para comprobar si tras la terapia se producía modificación de diferentes variables de personalidad, realizamos un Análisis de Varianza univariado mixto $2 \mathrm{x}(2)$, para cada una de las variables analizadas, teniendo el primer factor entre grupos independientes dos niveles (grupo terapia y grupo control) y un segundo factor intrasujeto de medidas repetidas con dos niveles (momento basal y momento post-terapia). Las variables dependientes fueron las puntuaciones obtenidas en las subescalas del NEO-FFI denominadas neuroticismo, extraversión, apertura a la experiencia, amabilidad y consciencia/responsabilidad; las puntuaciones en las subescalas de personalidad resistente denominadas compromiso o implicación, reto y control y por último las puntuaciones en optimismo. Los resultados mostraron que existían interacción entre momento x grupo en dos de las subescalas de personalidad del NEO-FFI, en concreto en la subescala de extraversión $[F(1,25)=5.84 ; p=.024]$ y consciencia/responsabilidad $[F(1,25)=4.70 ; p=.04]$. Por otro lado, también se encontró interacción en la variable disposicional optimismo $[F(1,25)=13.30 ; p<.001]$. No se encontró interacción en ninguna de las subescalas de personalidad resistente.

Por último, en el caso de las variables en las que existía interacción se llevaron a cabo diferentes $t$ de Student para muestras relacionadas con el objetivo de comprobar si había diferencias entre el momento preterapia y posterapia en cada uno de los grupos en estas variables. Los resultados encontrados, muestran que se produce un aumento del optimismo, la extraversión y la conciencia/responsabilidad en el grupo de tratamiento, produciéndose paralelamente una disminución en el grupo de control (Tabla 2 y Figura 1, 2 y 3). 
Tabla 1. Comparación de medias mediante $t$ de Student y tamaño del efecto entre el momento previo a la terapia y post terapia en ambos grupos.

\begin{tabular}{|c|c|c|c|c|c|c|}
\hline Escalas & Grupo & Pre-tratamiento & Post-tratamiento & $t$ & $p$ & Tamaño del efecto \\
\hline \multirow[t]{2}{*}{$\overline{\text { EEP }}$} & GT & $33.54(8.17)$ & $21.54(7.64)$ & 5.11 & $.000^{* *}$ & $1.52 * *$ \\
\hline & GC & $28.54(6.75)$ & $25.69(6.80)$ & 1.90 & .082 & .42 \\
\hline \multirow[t]{2}{*}{ VULNERAB-E } & GT & $13.62(4.54)$ & $8.00(6.03)$ & 4.24 & $.001 * *$ & $1.06^{* *}$ \\
\hline & GC & $10.69(5.59)$ & $8.54(5.90)$ & 3.90 & $.002 * *$ & .37 \\
\hline \multirow[t]{2}{*}{ PSWQ } & GT & $68.31(6.90)$ & $56.15(9.60)$ & 4.48 & $.001 * *$ & $1.47 * *$ \\
\hline & GC & $57.23(9.84)$ & $58.00(10.79)$ & -.32 & .751 & -.08 \\
\hline \multirow[t]{2}{*}{ SCL-SOM } & GT & $72.92(18.83)$ & $55.38(28.50)$ & 2.40 & $.034 *$ & $.741 *$ \\
\hline & GT & $63.92(13.52)$ & $65.46(20.05)$ & -.41 & .688 & -.09 \\
\hline \multirow[t]{2}{*}{ SCL-SI } & GT & 86.38 (19.94) & $55.54(29.18)$ & 4.67 & $.001 * *$ & $1.26 * *$ \\
\hline & GC & $66.23(20.60)$ & $57.69(21.29)$ & 1.53 & .153 & .41 \\
\hline \multirow[t]{2}{*}{ SCL-DEP } & GT & $77.77(20.38)$ & $49.54(30.17)$ & 3.96 & $.002 * *$ & $1.12^{* *}$ \\
\hline & GC & $68.46(22.93)$ & $61.50(22.70)$ & 2.19 & $.049 *$ & .31 \\
\hline \multirow[t]{2}{*}{ SCL-ANS } & GT & $78.00(16.88)$ & $51.23(18.86)$ & 4.18 & $.001 * *$ & $1.50 * *$ \\
\hline & GC & $61.31(17.24)$ & $60.58(20.41)$ & .28 & .787 & .04 \\
\hline \multirow[t]{2}{*}{ SCL-FOB } & GT & $40.54(37.32)$ & $36.77(30.07)$ & .33 & .747 & .11 \\
\hline & GC & $48.92(26.36)$ & $56.51(21.92)$ & -1.09 & .297 & -.31 \\
\hline
\end{tabular}

Nota: GT = grupo tratamiento, GC= grupo control. EEP = Escala de Estrés Percibido, VULNERAB-E = Inventario de Vulnerabilidad al Estrés, PSQW= Inventario de Preocupación, SCL= Inventario de Síntomas SCL-90-R, SOM= Somatizaciones, SI= Sensibilidad Interpersonal, DEP = Depresión, ANS= Ansiedad, FOB $=$ Ansiedad Fóbica.

Nota: $* * p<.02 ; * p<.05$. Tamaño del efecto medio (.6-.8)*; Tamaño del efecto alto $(>.8) * *$

Tabla 2. Comparación de medias mediante $t$ de Student y tamaño del efecto entre el momento previo a la terapia y post terapia en ambos grupos en variables de personalidad.

\begin{tabular}{|c|c|c|c|c|c|c|}
\hline Escalas & Grupo & Pre-tratamiento & Post-tratamiento & $t$ & $p$ & Tamaño del efecto \\
\hline \multirow[t]{2}{*}{ LOT } & GT & $12.08(3.57)$ & $14.92(3.59)$ & -3.75 & $.003 * *$ & $-.79 *$ \\
\hline & GC & $14.77(4.30)$ & $13.85(4.88)$ & 1.32 & .213 & .20 \\
\hline \multirow[t]{2}{*}{ NEO-E } & GT & $42.46(11.26)$ & $46.92(9.93)$ & -2.62 & $.022 *$ & -.42 \\
\hline & GC & $43.38(12.61)$ & $42.77(13.29)$ & .50 & .627 & .05 \\
\hline \multirow[t]{2}{*}{ NEO-C } & GT & 33.08 (13.24) & $37.23(9.77)$ & -1.35 & .203 & -.36 \\
\hline & GC & $42.77(12.44)$ & $38.08(8.54)$ & 1.75 & .105 & .45 \\
\hline
\end{tabular}

Nota: GT = grupo tratamiento, $\mathrm{GC}=$ grupo control. LOT $=$ Life Orientation Test, NEO= NEO-Five Factory Inventory, E $=$ Extraversión, $\mathrm{C}=\mathrm{Concien}-$ cia/Responsabilidad.

Nota: $* * p<.02 ; * p<.05$. Tamaño del efecto medio (.6-.8)*.

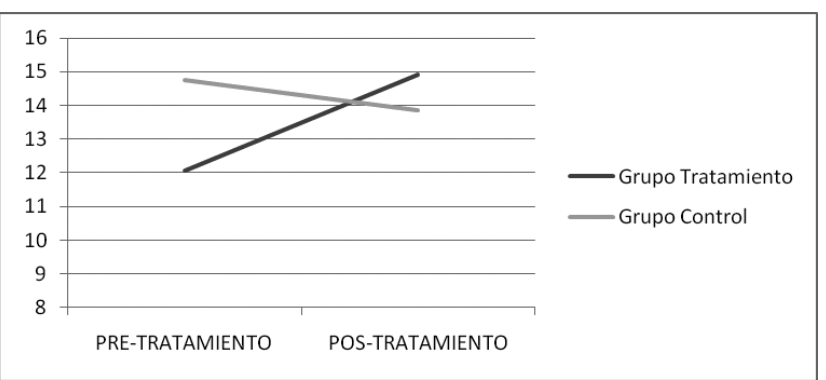

Figura 1. Puntuaciones en optimismo (LOT-R)

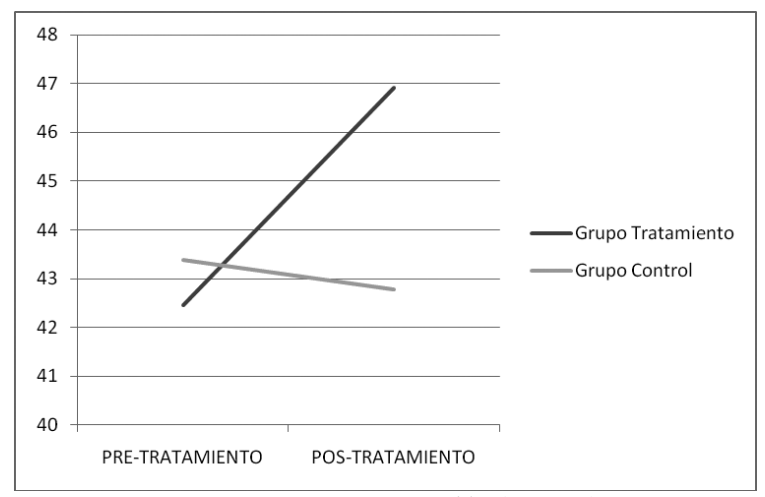

Figura 2. Factor extraversión (NEO E).

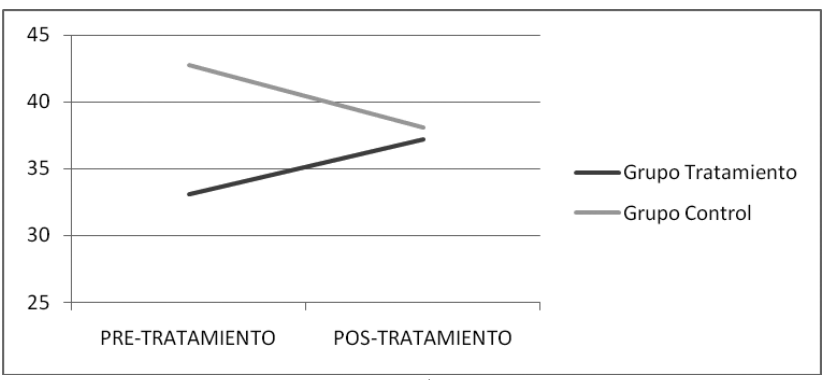

Figura 3. Factor conciencia/responsabilidad (NEO C).

\section{Discusión}

El objetivo del presente estudio fue comprobar si diferentes factores de personalidad relacionados con el estrés podían verse modificados mediante un programa para el control del estrés, basado en el uso de diversas herramientas cognitivoconductuales.

Los resultados encontrados mostraron por un lado que la terapia cognitivo-conductual de afrontamiento al estrés disminuyó las puntuaciones en las variables de estrés percibido, vulnerabilidad al estrés, preocupaciones, somatizaciones, sensibilidad interpersonal, depresión, ansiedad y ansiedad fóbica. Estos resultados confirman los hallazgos de 
otros estudios dirigidos a comprobar la eficacia de esta terapia para reducir los niveles de estrés y el malestar emocional (Navarrete-Navarrete et al., 2010; Peralta-Ramírez et al., 2009). Por otro lado, con respecto a las variables de personalidad también encontramos que tras la terapia se incrementan los niveles de optimismo, extraversión y conciencia/responsabilidad. Estos resultados tienen importantes implicaciones ya que se observa que con las herramientas psicológicas aportadas en el programa de control de estrés los participantes no solo mejoraron emocionalmente, resultados que eran esperables, sino que también cambiaron diferentes parámetros de su personalidad. En primer lugar, dado que el programa en su conjunto poseía como principal objetivo el control del estrés, éste se ve alcanzado a través de las puntuaciones en la Escala de Estrés Percibido. Así pues, los participantes informaron sobre la sensación subjetiva de poseer mayor control sobre las situaciones que anteriormente calificaban de estresantes, repercutiendo en las demás variables estudiadas en las que ya podemos ver cómo la puntuación en preocupaciones disminuye, al igual que en ansiedad y depresión, mejorando por tanto su estado emocional a nivel global. Hay que tener en cuenta que las situaciones que incitan a la preocupación seguían siendo las mismas para el grupo de terapia, sin embargo, entrenaron estrategias que les permitían controlarlas, impidiendo que éstas afectaran al funcionamiento de su vida cotidiana.

Con respecto a los resultados encontrados en las variables de personalidad hay que decir que el optimismo, el sentido de control y la habilidad para encontrar significados a las experiencias de la vida han sido siempre considerados como valiosos recursos psicológicos asociados a la salud mental, sin embargo también se presentan como elementos relevantes cuando las personas se hallan en situaciones que amenazan sus vidas o ante experiencias retantes (Albiñana, Sitges y Doménech, 2000). El cambio experimentado en optimismo por parte de la población tratamiento podría ser atribuible al contenido de las sesiones ya que dedicamos una sesión específica a optimismo y buen humor y dos sesiones a que las personas controlen su ira y hostilidad (emoción contraria a la alegría). Así mismo, también encontramos cambios importantes en la variable extraversión, este cambio podría deberse a que en el tratamiento hemos trabajado ampliamente la importancia del apoyo social y hemos dotado a los pacientes de las habilidades sociales más importantes de cara a enfrentarse a diferentes conflictos sociales. En esta línea en los estudios desarrollados por Martina y Castro (2003), el factor extraversión se vinculaba con la búsqueda activa del refuerzo en los otros (comunicatividad), presentando como meta motivacional la modificación activa del ambiente. Se encontraron relaciones importantes entre este factor y las características de la personalidad relacionadas con la asertividad, la confianza en sí mismo y la persuasión.

Además de las sesiones específicas, es importante subrayar que a lo largo de las 14 sesiones se ha complementado de forma transversal con reestructuración cognitiva. También es importante destacar que la población incluida en el estu- dio no es mayor, y esto hace que sea más fácil suavizar determinados patrones de personalidad ya que en poblaciones con edad más avanzada se hace más difícil la modulación de estos rasgos (Larsen y Buss, 2002).

Los estudios sobre cómo la personalidad se modifica tras una terapia son escasos (Benedito Moleón, Botella Arbona y López Andreu, 1996; De Fruyt, Van Leeuwen, Bagby, Rolland y Rouillon, 2006). Ello se debe a: a) la popularidad de los enfoques conductuales y de la estandarización de la medición de la personalidad; b) la creencia derivada de perspectivas humanistas que considera al diagnóstico y la evaluación como prácticas que no aportan nada a la relación de ayuda a las personas que buscan la psicoterapia; c) la creciente popularidad de los enfoques cognitivos con un énfasis en constructos acotados; d) la creencia respecto a que los test de personalidad no operan bien y cuentan con coeficientes de validez imprecisos debido a que centran su medición en los rasgos de personalidad, excluyendo las variables situacionales; e) el exceso de tiempo requerido para la medición y f) el énfasis actual en la realización de tratamientos breves, orientados a las crisis y cuyo objetivo es la restauración de los niveles anteriores de funcionamiento (Lambert y Supplee, 1997; Tomicic y Martínez, 2009). Los escasos estudios sobre los efectos de la terapia en la personalidad, fundamentalmente se han centrado en trastornos de la personalidad (Day y Bryan, 2007; Fonagy, Roth y Higgits, 2005).

Sin embargo, a pesar de la modificación de diversos factores de personalidad es importante hacer la apreciación de que la personalidad resistente no ha sufrido cambios en ninguno de los dos grupos. Al igual que hemos constatado el escaso número de investigaciones sobre el efecto de la terapia en el cambio de la personalidad (no patológica), los estudios sobre personalidad resistente se han centrado fundamentalmente en cómo la presencia de altos niveles de personalidad resistente se asocia a bajos niveles de estrés y burnout (Moreno-Jiménez et al., 2000; Moreno-Jiménez et al., 2006) sin embargo no conocemos estudios que se hayan dirigido a modificar los niveles de personalidad resistente. Por otra parte, una posible explicación a los resultados obtenidos podría ser que la muestra de nuestro estudio se ha caracterizado por altos niveles de personalidad resistente (puntuaciones medias entorno a 3 puntos, siendo el rango posible en el cuestionario de personalidad resistente utilizado entre 1 y 4) siendo difícil poder incrementar de forma estadísticamente significativa estos valores ya que en la línea base eran altos.

Hills y Norvell (1991) han encontrado que la personalidad resistente actuaba con un efecto principal más que modulador sobre el burnout y los síntomas físicos asociados. Por tanto, proponemos una nueva línea de investigación que persiga estudiar qué aspectos habría que incorporar en una terapia, con el fin de fomentar la personalidad resistente.

Nuestro estudio presenta una serie de limitaciones que deberían ser tenidas en cuenta para un futuro. En primer lugar, sería interesante que el número de participantes de cada grupo hubiera sido mayor ya que esto haría más consistentes 
los resultados encontrados. Por otro lado, un problema asociado es que todos los participantes del grupo terapia decidieron voluntariamente formar parte del programa debido al estrés que sentían, atribuyendo su situación de estrés a factores de la vida familiar, más que la propiamente académica o laboral. Frente a ello, el grupo control no solicitó ningún tipo de ayuda, de lo cual podemos interpretar que no se percibían con necesidad de apoyo psicológico por el estrés que experimentaban. Todo esto hace que aunque son grupos igualados en las principales variables psicológicas y emocionales, en otras variables no estaban igualados (como por ejemplo, ansiedad, hostilidad, sensibilidad interpersonal y nivel de preocupaciones) y partían de unas necesidades psicológicas diferentes. De este modo, una de las principales dificultades que se nos ha planteado en el diseño de esta investigación ha sido encontrar un grupo control homogéneo en

\section{Referencias}

Albiñana, P., Sitges, E. y Doménech, F. (2000). Relación entre percepciones de las características de personalidad y nivel de salud desde la perspectiva del sujeto. Revista de psicología de la salud, 12, 91-110.

Beech, H. R., Burns, L. E. y Scheffield, B. F. (1986). Tratamiento del estrés. Un enfoque comportamental. Madrid: Ed. Alambra.

Benedito Moleón, M. C., Botella Arbona, C. y López Andreu, J. A. (1996). Influencia de tres tratamiento psicológicos sobre dimensiones de personalidad en niños asmáticos. Anales de Psicología, 12, 217-222.

Brown, T. A., Antony, M. M. y Barlow, D. H. (1992). Psychometric properties of the Penn State Worry Questionnaire in a clinical anxiety disorders sample. Behaviour Research and Therapy, 30, 33-37.

Butler A. C., Chapman J. E., Forman E. M. y Beck A.T. (2006). The empirical status of cognitive-behavioral therapy: A review of metaanalyses. Clin Psychol Re, 26, 17-31.

Carver, C. S. y Scheier, M. F. (2002). Optimism. En Snyder y López. (Eds.), Handbook of Positive Psychology (pp. 231-243). New York: Oxford University Press.

Cohen, S., Kamarck T. y Mermelstein R. (1983). A global measure of perceived stress. Journal Health Social Behaviour, 24, 385-396.

Costa, P. T. y McCrae, R. R. (1992). Revised NEO Personality Inventory (NEOPIR) and NEO Five-Factor Inventory (NEO-FFI) professional manual. Odessa: Psychological Assessment Resources.

Day, A. y Bryan, J. (2007). Personality change and personality disorder: some initial thoughts on the application of McAdam's triarchic model to the treatment of personality disorder. Psychology, Crime \& Law, 13, 19-26.

De Fruyt, F., Van Leeuwen, K., Bagby, R. M., Rolland, J. y Rouillon, F. (2006). Assesing and interpreting personality change and continuity in patients trated for major depression. Psychological Assessment, 18(1), 71-80.

De Las Cuevas, C., González de Rivera, J. L., Henry, M., y Gracia, R. (1991) Análisis Factorial de la Versión Española del SCL-90-R en la Población General. Anales de Psiquiatría, 7, 93-96.

Denollet, J., de Jonge, P., Kuyper, A., Schene, A. H., van Melle, J.P., Ormel, J. y Honig, A. (2009). Depression and Type D personality represent different forms of distress in the Myocardial Infarction and DepressionIntervention Trial (MIND-IT). Psychological Medicine, 39, 749-756.

Derogatis, L. R. (1994). Symptom checklist 90. Administration Scoring and Procedures Manual. National Computer Systems Inc. Minneapolis

Ferrando, J., Chico, E. y Tous, J. (2002). Propiedades psicométricas del test de optimismo Life Orientation Test. Psicothema, 14, 673-680.

Fonagy, P., Roth, A., y Higgits, A. (2005). Psychodynamic psychotherapies: evidence-based practice and clinical wisdom. Bulletin of the Menninger Clinic, 69(1), 1-57.

Funk, S. C. (1992). Hardiness: a review of theory and research. Health Psychology, 11, 335-345.

Grases, G., Trías, C., Sánchez, C. y Zárate, J. (2010). Estudio del efecto del humor positivo como variable reductora del estrés y la ansiedad: Rela- variables y necesidades psicológicas al grupo terapia para comparar los efectos de participar en el programa o no hacerlo. Por último, subrayar que otra limitación del estudio ha sido no poder llevar a cabo un seguimiento de los participantes para comprobar el grado de estabilidad del cambio observado en las variables emocionales y de personalidad, lo que nos lleva afirmar que se producen cambios en factores de personalidad a corto plazo. En futuras investigaciones deberían considerar realizar un seguimiento para comprobar si este cambio desaparece con el tiempo o se estabiliza.

En conclusión, destacamos que los resultados encontrados nos informan de la posibilidad de modificar diferentes variables de personalidad que tradicionalmente se consideraban estables, mejorando con ello el abordaje psicológico y emocional que la persona hace ante las continuas situaciones de estrés cotidiano que experimenta.

ción del humor con variables de personalidad y rendimiento. Apuntes de Psicología, 28, 143-163.

Hills, H. y Norvell, N. (1991). An examination of hardiness and neurocitism as potencial moderators of stress outcomes. Behavioral Medicine, 17, 3138.

Ho, R. C., Neo, L. F., Chua, A. N., Cheak, A. A. y Mak, A. (2010). Research on psychoneuroimmunology: does stress influence immunity and cause coronary artery disease? Ann Acad Med Singapore, 39, 191-196.

Hofmann, S. G. y Smits, J. A. J. (2008). Cognitive-behavioral therapy for adult anxiety disorders: a meta-analysis of randomized placebocontrolled trials. Journal of Clinical Psychiatry, 69, 621-632.

Hombrados, M. I. (1997). Estrés y salud. Valencia: Promolibro.

Kobasa, S. (1979). Stressful live events personality and health an inquiri into hardiness. Journal of Personality and social Psychology, 37, 1-11.

Kobasa, S. C. (1982). The hardy personality: toward a social psychology of stress and health. En G. S. Sanders y J. Suls (Eds.), Social psychology of bealth and illness. Hillsdale, N. J. Lawrence Erlbaum Associates, Inc.

Kobasa, S., Maddi, S. y Kahn, S. (1982). Hardiness and health: A prospective study. Journal of Personality and Social Psychology, 42, 168-177.

Lambert, M. y Supplee, E. (1997). Trends and practice in psychotherapy outcome assessment and their implicatios for psychotherapy and applied personality. En R. Hogan, J. Johnson y S. Briggs. (Eds.), Handbook of personality psychology. New York: Academic Press.

Larsen, R. J. y Buss, D. M. (2002). Personality Psychology: domains of knowledge about nature, 2nd ed. McGraw Hill.

Lazarus, R. S. y Folkman, S. (1986). Estrés y procesos cognitivos. Barcelona: Martínez Roca.

Lecic-Tosevski, D., Vukovic, O., Stepanovic, J. (2011). Stress and personality. Psychiatrike, 22, 290-7.

López, A. F., Kasanzew, A. y López, M. B. (2007). Efectos terapéuticos positivos a través del aumento del optimismo. Ciencias Psicológicas, 1, 179188.

Lyubomirsky, S., King, L. y Diener, E. (2005). The benefits of frequent positive affect: Does happiness lead to success? Psychological Bulletin, 131, 803-855.

Manga, D., Ramos, F. y Morán, C. (2004). The Spanish norms of the NEO Five-Factor Inventory: New data and analyses for its improvement. International Journal of Psychology and Psychological Therapy, 4, 639-348.

Martina, M. y Castro, A. (2003). Factores y estilo de personalidad. Estudio comparativo. Avaliação Psicológica, 1, 35-43.

Martínez-Correa, A., Reyes del Paso, G. A., García-León, A. y GonzálezJareño, M.I. (2006) Optimismo/pesimismo disposicional y estrategias de afrontamiento del estrés. Psicothema, 18 (1) 66-71

Meyer, T. J., Miller, M. L., Metzger, R. L. y Borkovec, T. D. (1990). Development and validation of the Penn State Worry Questionnaire. Behaviour Research and Therapy, 28, 487-495. 
Moreno-Jiménez, B., Garrosa Hernández, E. y González Gutiérrez, J. L. (2000). Personalidad resistente, burnout y salud. Escritos de Psicología, 4, 64-77.

Moreno Jiménez, B., Morett Natera, N. I., Rodríguez Muñoz, A. y Morante Benadero, M. E. (2006) La personalidad resistente como variable moduladora del síndrome de burnout en una muestra de bomberos. Psicothe$m a, 18$ (3), 4-13-418.

Navarrete-Navarrete, N., Peralta-Ramírez, M. I., Sabio-Sánchez, J. M., Coín, M. A., Robles-Ortega, H., Hidalgo-Tenorio, C., Ortego-Centeno, N., Callejas-Rubio, J. L. y Jiménez-Alonso, J. (2010). Effects of the treatment of chronic stress in patients with lupus erythematosus: a randomized controlled trial. Psychosomatics and Psychotherapy, 79, 107-115.

Nes, L. S. y Segerstrom, S. C. (2006). Dispositional optimism and coping: a meta-analytic review. Personality and Social Psychology Review, 10, 235-51.

Öst, L. G. (2008). Cognitive behavior therapy for anxiety disorders: 40 years of progress. Nordic Journal of Psychiatry, 62, 5-10.

Ožura, A., Ihan, A. y Musek, J. (2012). Can the big five factors of personality predict lymphocyte counts? Psychiatria Danubina, 24, 66-72.

Padilla Montemayor, V. M., Peña Moreno, J. A. y Arriaga, A. E. (2006). Patrones de personalidad tipo A o B, estrés laboral y correlatos psicofisiológicos. Psicología y Salud, 16, 79-85.

Peralta-Ramírez, M. I., Robles-Ortega, H., Navarrete-Navarrete, N, y Jiménez-Alonso, J. (2009). Aplicación de la terapia de afrontamiento al estrés en dos poblaciones con alto estrés: pacientes crónicos y personas sanas. Salud Mental, 32, 251-258.

Remor E. (2006) Psychometric Properties of a European Spanish Version of the Perceived Stress Scale (PSS). Spanish Journal of Psychology, 9, 86-93.

Remor, E. y Carrobles, J.A. (2001). Versión española de la Escala de Estrés Percibido (PSS-14): estudio psicométrico en una muestra de VIH+. Ansiedad y estrés, 7, 195-201.

Riolli, L. y Savicki, V. (2003). Optimism and doping moderators of the relation between work resources and burnout in information services workers. International Journal of Stress Management, 10, 235-252.

Ríos Rísquez, M. I., Godoy Fernández, C. y Sánchez-Meca, J. (2011). Síndrome de quemarse por el trabajo, personalidad resistente y malestar psicológico en personal de enfermería. Anales de Psicología, 27, 71-79

Robles Ortega, H. y Peralta Ramírez, M. I. (2006) Programa para el control del estrés. Madrid: Ediciones Pirámide.
Robles-Ortega, H., Peralta-Ramírez, M. I. y Navarrete-Navarrete, N. (2006). Validación de la versión española del Inventario de Vulnerabilidad al Estrés de Beech, Burns y Scheffield. Avances en Psicología de la Salud (p.62). Granada: Sider.

Sandín, B. (2003). El estrés: un análisis basado en el papel de los factores sociales. Revista Internacional de Psicología Clínica y de la Salud, 3, 141-157.

Sandín-Ferrero, B., Chorot-Raso, P., Valiente-García, R. y Lostao-Unzu, L. (2009). Validación española del cuestionario de preocupación PSWQ: estructura factorial y propiedades psicométricas (Spahish validation of the PSWQ: factor structure and psychometric properties). Revista de Psicopatología y Psicología Clinica, 14(2), 107-122.

Santos-Ruiz, A., García-Ríos, M. C., Fernández-Sánchez, J. C., Pérez-García, M., Muñoz-García, M. A. y Peralta-Ramírez, M. I. (2012). Can decision-making skills affect responses to psychological stress in healthy women? Psychoneuroendocrinology, 37, 1912-21.

Sapolsky, R. M. (2008). ¿Por qué las cebras no tienen úlcera? La guía del estrés. Madrid: Alianza Editorial.

Scheier, M. F., Carver, C. S. y Bridges, M. W. (1994). Distinguishing optimism from neuroticism (and trait anxiety, self-mastery, and self-steem): a reevaluation of Life Orientation Test. Journal of Personality and Social Psychology, 67, 1063-1078.

Schiffrin, H. y Nelson, K. (2010) Stressed and Happy Investigating the Relationship Between Happiness and Perceived Stress. Journal of Happines. Studies, 11, 33-39.

Schou, I., Ekeberg, O., Ruland, C. M., Sandwik, L. y Karesen, R. (2004). Pessimism as a predictor of emotional morbidity one year following breast cancer surgery. Psycho-oncology, 13, 309-320.

Shifren, K. y Hooker, K. (1995). Stability and change in optimism: A study among spouse caregivers. Experimental Aging Research, 21, 59-76.

Spangler, D., Simons, A., Monroe, S. y Thase, M. (1997). Response to Cognitive-Behavioral Therapy in Depression: Effects of Pretreatment Cognitive Dysfunction and Life Stress. Journal of Consulting \& Clinical Psychology, 65, 568-575.

Tomicic, A. y Martínez, C. (2009). La Variable Personalidad en el Estudio de la Psicoterapia: ¿Medida de Resultado o Proceso? Terapia Psicológica, 27, 51-60.

(Articulo recibido: 12-09-2011; revisado: 01-07-2013; aceptado: 27-07-2013) 